\title{
Presence of mechanical dyssynchrony in duchenne muscular dystrophy
}

\author{
Kan N Hor ${ }^{1}$, Janaka P Wansapura², Hussein R Al-Khalidi', William M Gottliebson', Michael D Taylor ${ }^{1}$, Richard J Czosek', \\ Sherif F Nagueh ${ }^{4}$, Nandakishore Akula', Eugene S Chung ${ }^{5}$, Woodrow D Benson ${ }^{1}$, Wojciech Mazur ${ }^{5^{*}}$
}

\begin{abstract}
Background: Cardiac dysfunction in boys with Duchenne muscular dystrophy (DMD) is a leading cause of death. Cardiac resynchronization therapy (CRT) has been shown to dramatically decrease mortality in eligible adult population with congestive heart failure. We hypothesized that mechanical dyssynchrony is present in DMD patients and that cardiovascular magnetic resonance (CMR) may predict CRT efficacy.

Methods: DMD patients $(n=236)$ were stratified into 4 groups based on age, diagnosis of DMD, left ventricular (LV) ejection fraction (EF), and presence of myocardial fibrosis defined as positive late gadolinum enhancement (LGE) compared to normal controls $(n=77)$. Dyssynchrony indices were calculated based on timing of CMR derived circumferential strain ( $\left.e_{c c}\right)$. The calculated indices included cross-correlation delay $(X C D)$, uniformity of strain (US), regional vector of variance (RW), time to maximum strain (TTMS) and standard deviation (SD) of TTMS. Abnormal XCD value was defined as > normal + 2SD. US, RW, TTMS and SD were calculated for patients with abnormal XCD.

Results: There was overall low prevalence of circumferential dyssynchrony in the entire DMD population; it increased to $17.1 \%$ for patients with abnormal EF and to $31.2 \%$ in the most advanced stage (abnormal EF with fibrosis). All but one DMD patient with mechanical dyssynchrony exhibited normal QRS duration suggesting absence of electrical dyssynchrony. The calculated US and RW values $(0.91 \pm 0.09,1.34 \pm 0.48)$ indicate disperse rather than clustered dyssynchrony.
\end{abstract}

Conclusion: Mechanical dyssynchrony is frequent in boys with end stage DMD-associated cardiac dysfunction. It is associated with normal QRS complex as well as extensive lateral fibrosis. Based on these findings, it is unlikely that this patient population will benefit from CRT.

\section{Background}

In patients with New York Heart Association (NYHA) class III, ambulatory class IV systolic heart failure (HF) and recently class I and II, with electrocardiographic evidence of ventricular dyssynchrony, cardiac resynchronization therapy (CRT) improves quality of life and functional status, reduces heart failure-related hospitalizations, and prolongs survival [1-4]. While current indications are based on QRS duration > $120 \mathrm{~ms}$, HF patients with narrow QRS complexes have been investigated for the presence of mechanical dyssynchrony. In this population, the prevalence of dyssynchrony is

\footnotetext{
* Correspondence: mazurw@ohioheart.org

${ }^{5}$ The Heart and Vascular Center, The Christ Hospital, Cincinnati, Ohio, USA Full list of author information is available at the end of the article
}

present though at a lower frequency compared to patients with prolonged QRS. Accordingly, in several small pilot studies [5-8] these patients appear to benefit from CRT, although a large multicenter randomized trial did not meet its primary endpoint [9]. In the pediatric heart failure population there is even less data and there are no large retrospective or prospective trials in reference to CRT [10].

A major cause of pediatric HF, Duchenne muscular dystrophy (DMD) is an X-linked recessive disorder characterized by disruption of the dystrophin protein. The resulting cell membrane degradation in skeletal and cardiac muscle tissue [11-13] leads to a cascade of inflammation, edema, necrosis and fatty replacement [14-17]. This ultimately results in the development of myocardial fibrosis. Although the extent of involvement varies,
C Biomed Central

() 2011 Hor et al; licensee BioMed Central Ltd. This is an Open Access article distributed under the terms of the Creative Commons Attribution License (http://creativecommons.org/licenses/by/2.0), which permits unrestricted use, distribution, and reproduction in any medium, provided the original work is properly cited. 
overt cardiac systolic dysfunction is present by 20 years of age in essentially all DMD patients. Current therapeutic options for DMD associated HF are limited with no established standard of care for medical or device interventions. This study sought to determine the feasibility of CRT in the DMD population, most of whom have normal QRS complexes. We evaluated the prevalence and character of mechanical dyssynchrony using the cross correlation delay (XCD) method, a highly objective, automatable dyssynchrony parameter on tagged cardiovascular magnetic resonance (CMR) [18].

\section{Methods}

\section{Study Population}

This is a retrospective cohort analysis and was conducted with the approval of the Institutional Review Board at the Cincinnati Children's Hospital Medical Center. Patients were identified using the clinical CMR database at the Heart Institute at Cincinnati Children's Hospital Medical Center from September 2005 to March 2009. This study included all DMD patients > 7 years of age who had previously undergone clinical CMR studies. Subjects $<7$ years of age were excluded in order to eliminate the need for sedation to undergo the CMR study. A diagnosis of DMD was confirmed by a skeletal muscle biopsy and/or a DNA analysis demonstrating a characteristic dystrophin mutation.

Genetic testing was performed at Athena Diagnostics, Inc. or Medical Genetics Laboratories, Baylor College of Medicine (prior to 2006) and at Emory University Genetics Laboratory, Atlanta, Georgia (since 2006).

\section{Subject groups}

DMD patients $(\mathrm{n}=236)$ were stratified into 4 groups based on age, left ventricular (LV) ejection fraction (EF), presence of myocardial fibrosis defined as positive late gadolinum enhancement (LGE) and compared to normal controls (Figure 1).

\section{CMR and HARP ${ }^{\circledR}$ Analyses}

Clinical CMR studies were conducted on either a Siemens 3 Tesla Trio (Siemens Medical Solutions, Malvern, PA/Erlangen, Germany) or on a 1.5 Tesla GE Signa Excite (General Electric Healthcare; Milwaukee, WI). The type and field strength of MRI system was based solely on clinical availability and scheduled independent of the patient's clinical status or prior type or field strength of the first study.

Routine cardiac functional imaging was performed with a retrospectively vectorcardiographic (VCG) gated, segmented Steady State Free Precession (SSFP) technique after localized shimming and/or frequency adjusting. Subjects were breath-held as tolerated; for those subjects who could not adequately breath-hold, a free breathing technique with multiple signal averaging was used. Standard functional imaging included a short axis stack of cine SSFP images from cardiac base to apex; the short axis was prescribed as the perpendicular plane to the left ventricular long axis in 2 and 4 chamber views as per previously published protocols. Typical scan parameters were as follows: $\mathrm{FOV}=32-38 \mathrm{~cm}$, slice thickness $=$ 5-6 mm, gap = 1-2 mm, NEX = 2 (breath hold; 4-5 for free breathing), $\mathrm{TE} / \mathrm{TR}=1.4 / 2.8$ (Siemens), $\mathrm{TE} / \mathrm{TR}=2.0 /$ $4.0(\mathrm{GE})$, in-plane resolution $=1.2-2.2 \mathrm{~mm}$. Minimums of 12 slices were performed with 20 phases/slice.

Tagged cine CMR images were acquired in the short axis of the mid ventricle at the level of the papillary muscles based on the four chamber view using an ECGtriggered segmented k-space fast gradient echo sequence with spatial modulation of magnetization in orthogonal planes. Grid tag spacing was $8 \mathrm{~mm}$. The scan parameters used were: $\mathrm{TE} / \mathrm{TR}=3 / 15 \mathrm{~ms}$. field of view $=(30-32) \times$ $(25-26) \mathrm{cm}$, slice thickness $=6 \mathrm{~mm}$, acquisition matrix $=$ $256 \times 144$, flip $=20^{\circ}$, views per segments $=8-9$. Only good quality tagged cine MRIs were included for analysis (confirmed by 2 independent expert readers: NA and $\mathrm{KNH}$ ).

\section{Late gadolinum enhancement (LGE)}

LGE imaging was performed on DMD patients when intravenous access was obtained $(\mathrm{n}=121)$; no LGE imaging was performed in the control group. LGE imaging was performed via a FLASH inversion sequence recovery protocol $10 \mathrm{~min}$ after $0.2 \mathrm{mmol} / \mathrm{kg}$ gadolinium diethylenetriamine penta-acetic acid (Gd-DTPA) injection. LGE imaging was considered positive if any area of the mid-myocardium showed hyperenhancement as assessed by consensus of 3 readers (KNH, WMG, WM)

\section{ECG Analysis}

Standard 12 lead ECG was obtained in all patients; QRS duration was measured manually.

\section{Data Analysis}

\section{Left ventricular function and volumes}

Left ventricular volumes, mass and global function were assessed via standard planimetry techniques using semi automated computer software (QMASS v.6.1.5, Medis Medical Imaging Systems, Netherlands) by expert readers (KNH and WMG). Left ventricular stroke volume was also determined by analysis of cine velocity encoded phase contrast gradient echo (GRE) sequence of the ascending aorta via Medis QFLOW $^{\circledR}$ platforms for internal validation of the stroke volume determined by ventricular planimetry. Ventricular volumes, mass, and ejection fraction were tabulated for each subject.

\section{Myocardial Strain Analysis}

As previously described, tagged images from two times points of the mid-ventricle were analyzed using the 


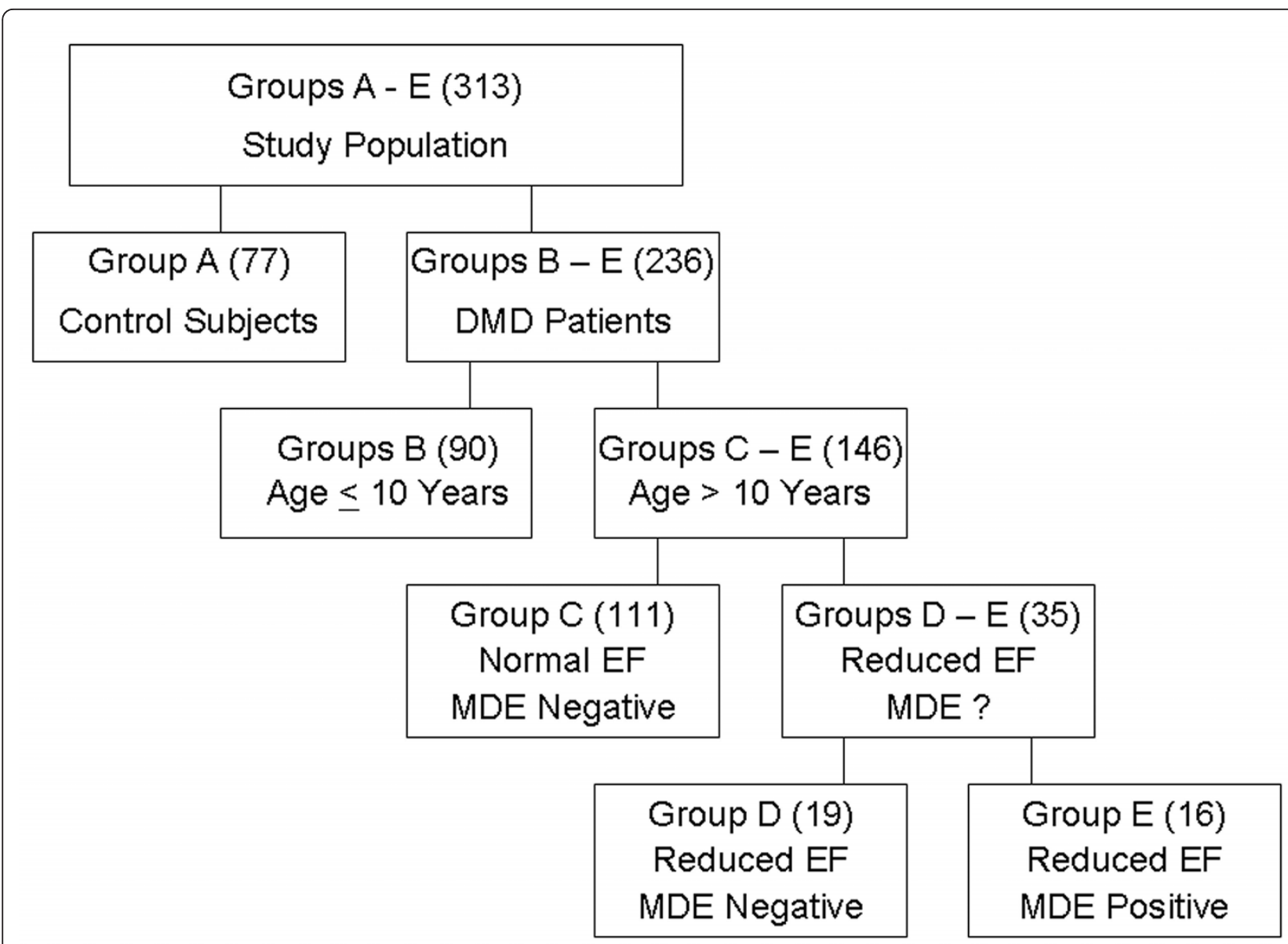

Figure 1 Group Stratification. DMD patients $(n=236)$ were stratified into 4 groups based on age, diagnosis of DMD, left ventricular (LV) ejection fraction (EF), and presence of myocardial fibrosis defined as positive late gadolinium enhancement(LGE). DMD and compared to normal controls.

HARmonic Phase (HARP, Diagnosoft, CA, USA) technique [19-23]. Details of circumferential strain $\left(\varepsilon_{\text {cc }}\right)$ analysis have been previously described $[24,25]$. Only the mid-ventricular slice was analyzed secondary to $[24,25]$ the limited reproducibility of the analysis of the basal and apical slices. The regional (absolute value and time to peak) and global $\varepsilon_{\mathrm{cc}}$ data was exported to a spreadsheet file for analysis.

\section{Late Gadolinum Enhancement Analysis}

LGE transmural extent was calculated as the ratio of myocardial LGE thickness to total wall thickness. It was expressed in 2 categories: $<50 \%$, and $>50 \%$.

\section{Dyssynchrony Assessment}

$\varepsilon_{\mathrm{cc}}$ vs. time curves from 24 sub-regions at the mid ventricular level were used. Six regional $\varepsilon_{\mathrm{cc}}$ curves were constructed by taking the average of four adjacent subregions to represent the segments 7-12 in the AHA 17 segment heart model. Sub-regional $\varepsilon_{\mathrm{cc}}$ curves were used in the analysis of spatial dyssynchrony quantified by Uniformity of Strain (US), and Regional Vector of
Variance (RVV). Regional $\varepsilon_{\mathrm{cc}}$ curves were used in the analysis of temporal dyssynchrony quantified by crosscorrelation delay (XCD), time to maximum strain (TTMS) and the standard deviation of TTMS (STD). Data were analyzed using custom developed software using IDL (IDL 6.2, ITT Visual Information Solutions, Boulder, $\mathrm{CO}$ ).

(1) The cross-correlation delay (XCD)(18) between two curves was computed by shifting one curve in time relative to the other curve and computing the normalized correlation between the curves for each time shift. The time shift between the two curves that resulted in the maximum correlation value was de冈ned as the temporal delay between the two curves. A temporal delay was computed between the anterior and inferior segments (XCD 7-10), the anteroseptal and inferolateral segments, (XCD 8-11) and the inferoseptal and anterolateral segments, (XCD 9-12) and the reported XCD was the maximum of these three delays. As normal values for above variables have not been published, we used 
values $\geq$ mean +2 standard deviations for XCD as indicative for dyssynchrony.

(2) Time to maximum strain (TTMS) was calculated as follows: for each regional $\varepsilon_{\mathrm{cc}}$ curve, the time from the onset of QRS to the maximum absolute $\varepsilon_{\mathrm{cc}}\left(\mathrm{T}_{\max }\right)$ was determined. Then TTMS was calculated as the difference between the shortest and the longest $\mathrm{T}_{\max }$ i.e. the time difference between the earliest and the latest time at which a region reaches its maximum strain.

(3) The standard deviation (STD) of $T_{\max }$ is calculated for each of the six regions.

In addition to the above, we evaluated two additional indices of "global dyssynchrony" differentiating between "clustered" and "dispersed "dyssynchrony (Figure 2) [26].

(1) Uniformity of Strain (US) [27] was derived by subjecting the spatial plot of sub-regional strains at end systole (defined by the time at which mean $\varepsilon_{\mathrm{cc}}$ reached maximum) to Fourier analysis. If all sub-regions shorten simultaneously (synchronously), the plot appears as a straight line, with power only in the zero-order Fourier term $\left(A_{0}\right)$, whereas regionally clustered dyssynchrony generates an undulating plot with higher power in the first-order term $\left(A_{1}\right)$. The US index is defined as $\sqrt{A_{0}^{2} /\left(A_{0}^{2}+2 A_{1}^{2}\right)}$. The US index is zero for dyssynchronous contraction and 1 for perfectly synchronous contraction.

(2) Regional vector of variance (RVV) [28] was derived by representing the $\varepsilon_{\mathrm{cc}}$ value of the $\mathrm{n}^{\text {th }}$ sub-region at a given time point by a vector with magnitude $\varepsilon_{\mathrm{cc}}(\mathrm{n})$ and phase $2 \pi n / 24,(n=1 . .24)$. RVV was calculated as the vector sum of all $\varepsilon_{\mathrm{cc}}(\mathrm{n})$ giving a zero value for perfect synchrony. The maximum value of RVV depends on the number of segments analyzed. For 24 segments the maximum RVV value is 7.66 .
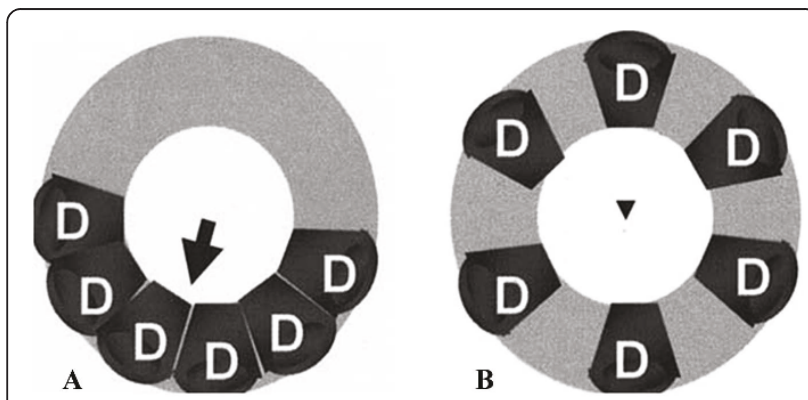

Figure 2 Schematic representation of clustered (A) and disperse (B) dyssynchrony. Depending how dyssynchrony is being indexed, Results could be the same for both situations, despite markedly different impact on dsyynchrony. In example $B$, there is a "net" synchronous contraction. Uniformity of Strain (US) and Regional Variance of Strain (RW) are vector derived indices differentiating clustered from dispersed dyssynchrony. Adapted from: Lardo, A. C. et al. J Am Coll Cardiol 2005;46:2223-2228.
Patients with abnormal XCD were presumed to have mechanical dyssynchrony and TTMS, SD, RVV, US were also calculated and compared for groups A-E.

\section{Statistical Analysis}

Results are expressed as mean \pm SD for continuous data and as percentages and numbers for categorical data. Pearson's correlation coefficients were calculated to assess for linear relationships between relevant variables. Dunnett test was used to compare groups (B-E) to the control group (A) on variables of interest.

\section{Results}

236 consecutive patients with DMD where evaluated. Patient demographic, EF, mass volume ratio and mean values for all dyssynchrony indices are presented in Table 1. All the normal controls had absolute $\varepsilon_{\mathrm{cc}}$ greater than 17 and all DMD patients had absolute $\varepsilon_{\mathrm{cc}}$ less than 17. In DMD patients but not normal controls, strain declined with age as previously described [24].

LGE was observed in group E only, involving anterolateral and inferolateral segments, with extent of enhancement over $50 \%$ of wall thickness (Figure 3).

\section{QRS duration}

Summary statistics for QRS duration by groups are given in Table 1. Neither left nor right bundle branch block morphology was observed in our study group. There were 3 boys ( 1 in each group A, D and E) with QRS duration over $120 \mathrm{~ms}$ consistent with nonspecific intraventricular conduction delay.

\section{Dyssynchrony}

Comparison of boys with identified dyssynchrony to control group is presented in Table 2. We defined XCD to be the largest of the three XCD (XCD 7-10, XCD 8-11 or XCD 9-12) measurements. There were $9(3.8 \%)$ boys ( 3 in group C, 1 in group D and 5 in group E) with abnormal XCD, defined as XCD larger than $79.4 \mathrm{~ms}$ (calculated as mean + 2SD of XCD of normal control) In these 9 patients, TTMS was $111.2 \pm 39.5 \mathrm{~ms}$, RVV was $1.3 \pm 0.5$, STD was $45.6 \pm 18.1$, US was $0.9 \pm$ 0.10 and QRS duration was $98.2 \pm 15.3 \mathrm{~ms}$. Only 1 of those 9 boys had a QRS > $120 \mathrm{~ms}$. One-way ANOVA model comparing dyssynchrony indices in 9 DMD boys with mechanical dyssynchrony to those of control group A $(n=77)$ showed statistically significant differences in all indices except for QRS duration $(p=0.2825)$. In addition, RVV index was borderline significant ( $\mathrm{p}=$ 0.0538 ) as compared to the control group. It needs to be noted that despite statistical differences US and RVV values in boys with dyssynchrony are suggestive of nearly "net" synchronous contraction (disperse rather than clustered dyssynchrony) (Figure 2). 
Table 1 General Characteristic by Group

\begin{tabular}{|c|c|c|c|c|c|}
\hline \multirow[t]{2}{*}{ Parameter } & \multicolumn{5}{|l|}{ Group } \\
\hline & $\begin{array}{l}A \\
(n=77)\end{array}$ & $\begin{array}{l}B \\
(n=90)\end{array}$ & $\begin{array}{l}C \\
(n=111)\end{array}$ & $\begin{array}{l}D \\
(n=19)\end{array}$ & $\begin{array}{l}E \\
(n=16)\end{array}$ \\
\hline Age & $13.9 \pm 8.9$ & $8.5 \pm 0.9^{* *}$ & $12.9 \pm 2.8$ & $15.0 \pm 3.9$ & $17.3 \pm 5.4^{* *}$ \\
\hline LVEF (\%) & $64.6 \pm 5.9$ & $65.0 \pm 4.8$ & $64.4 \pm 5.8$ & $49.4 \pm 6.6^{* *}$ & $36.5 \pm 12.2^{* *}$ \\
\hline$X C D^{a}$ & $45.6 \pm 16.9$ & $35.3 \pm 16.6$ & $38.2 \pm 19.8$ & $52.2 \pm 22.4$ & $73.0 \pm 41.9$ \\
\hline US_max & $0.97 \pm 0.04$ & $0.98 \pm 0.03$ & $0.97 \pm 0.03$ & $0.97 \pm 0.04$ & $0.92 \pm 0.08^{* *}$ \\
\hline TTMS & $78.2 \pm 31.8$ & $65.4 \pm 22.9^{* *}$ & $71.2 \pm 24.0$ & $95.1 \pm 19.6^{* *}$ & $111.4 \pm 33.0^{* *}$ \\
\hline RW_max & $0.95 \pm 0.57$ & $1.03 \pm 0.55$ & $1.13 \pm 0.57$ & $1.03 \pm 0.51$ & $1.34 \pm 0.57^{* *}$ \\
\hline STD_peak & $31.7 \pm 12.0$ & $26.7 \pm 8.4^{* *}$ & $29.6 \pm 9.7$ & $36.5 \pm 6.9$ & $46.1 \pm 16.6^{* *}$ \\
\hline QRS & $92.8 \pm 11.4^{1}$ & $85.1 \pm 7.8^{* *}$ & $86.6 \pm 8.0^{* *}$ & $86.1 \pm 13.4$ & $97.1 \pm 12.9$ \\
\hline$\varepsilon_{\subset \subset}$ & $-18.2 \pm 4.5$ & $-14.2 \pm 1.4^{* *}$ & $-13.2 \pm 2.0^{* *}$ & $-10.7 \pm 2.1$ & $-7.0 \pm 2.8$ \\
\hline
\end{tabular}

\section{Discussion}

This study demonstrated that there is a significant prevalence of mechanical ventricular dyssynchrony (17\%) in DMD patients with $\mathrm{EF}<55 \%$. Additionally, the prevalence of dyssynchrony was greater in patient subsets with more advanced cardiac disease (low EF and fibrosis).

This is the first study to our knowledge to use an automated MRI derived cross correlation technique for assessment of mechanical dyssynchrony. It is also the first study to assess prevalence and characteristics of dyssynchrony in a large DMD patient population. Previous application of the XCD method utilized echocardiography [29] data. Subsequently, CMR (phase contrast) [30,31] was found to be superior for measuring time to peak dyssynchrony parameters for discriminating between subjects with and without dyssynchrony. Circumferential strain is an established CMR method of dyssynchrony assessment, Han et al evaluated circumferential myocardial strain in cardiomyopathy with and without left bundle branch block; these authors assessed opposing wall delays, and reported values within the range of XCD values in our patient population [32]. The current study, to our knowledge is also the first to assess

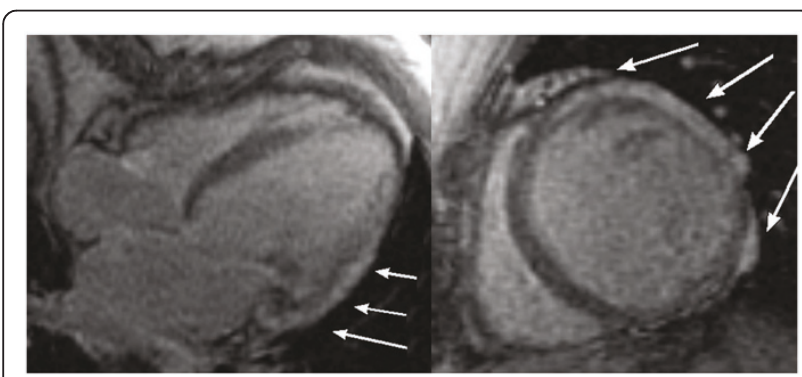

Figure 3 Late gadolinum enhancement (LGE). LGE images of a representative patient from group E. Note predominantly transmural enhancement involving anterolateral and inferolateral segments in apical four chambers and short axis views. circumferential dyssynchrony by XCD in a narrow QRS patient population.

Boys with DMD do not fulfil classic adult derived criteria for CRT: QRS complex is narrow in $97 \%$ of the population, EF is generally greater than $35 \%$, and due to the nature of the disease, NYHA functional class could not be assessed. These barriers, combined with compelling and devastating disease progression, make it even more important to develop sensitive tools to create prediction models as to likelihood of success with CRT in all or a distinct subset of this patient population. In this study we demonstrated a low prevalence of dyssynchrony in the overall population of boys with DMD using cross-correlation delay; but the prevalence increased to $17.1 \%$ for patients with abnormal EF (groups D and E) and to $31.2 \%$ in the most advanced stage (group E). All but one of the boys with DMD and mechanical dyssynchrony exhibited normal QRS duration suggesting absence of electrical dyssynchrony. Only weak relationships were found between dyssynchrony

Table 2 Abnormal XCDs vs. the control

\begin{tabular}{lll}
\hline Parameter & \multicolumn{2}{l}{ Group } \\
\cline { 2 - 3 } & $\begin{array}{l}\text { Control } \\
(\mathbf{n}=\mathbf{7 7})\end{array}$ & $\begin{array}{l}\text { Abnormal XCDs } \\
(\mathbf{n}=\mathbf{9})\end{array}$ \\
\hline Age & $13.9 \pm 8.9$ & $16.7 \pm 5.0$ \\
\hline LVEF (\%) & $64.6 \pm 5.9$ & $43.9 \pm 16.7^{* *}$ \\
(min, max) & & \\
\hline LVRI & $0.71 \pm 0.19$ & $0.66 \pm 0.21$ \\
\hline XCD & $45.6 \pm 16.9$ & $116.5 \pm 20.5^{* *}$ \\
\hline Presence of LGE & $0(0.0 \%)$ & $4(57.1 \%)^{* *}$ \\
\hline US_max & $0.97 \pm 0.04$ & $0.91 \pm 0.09^{* *}$ \\
\hline TTMS & $78.2 \pm 31.8$ & $111.2 \pm 39.5^{* *}$ \\
\hline RW_max & $0.95 \pm 0.57$ & $1.34 \pm 0.48$ \\
\hline STD_peak & $31.7 \pm 12.0$ & $47.6 \pm 18.1^{* *}$ \\
\hline QRS & $92.8 \pm 11.4^{1}$ & $98.2 \pm 15.3$ \\
\hline$* * P<0.05$ as compared to the control group (A) & using Dunnett test. \\
${ }^{a}$ XCD = maximum of (XCD $1-4$, XCD 2-5 and XCD 3-6).
\end{tabular}


indices and $\varepsilon_{\mathrm{cc}}$ or EF likely due to low number of boys with low EF. Of interest, in those with dyssynchrony, US and RVV values indicate the presence of disperse rather than clustered dyssynchrony. Disperse dyssynchrony patients would not be expected to respond favourably to CRT (Figure 2).

Location of scar tissue is concerning as well, as most common LGE location was in the inferolateral segments. In patients with ischemic CMP, inferolateral scar involving over $50 \%$ of wall thickness virtually excluded improvement with CRT [33], as did total scar volume of over $15 \%[34,35]$. We could not assess total scar volume in our study as only 3 short axis slices were acquired for LGE assessment. Even if left ventricular lead was implanted before the development of extensive inferolateral fibrosis, it is possible that disease progression in that segment would diminish efficacy of CRT over time.

Do these barriers preclude the possibility that LV pacing might benefit these patients? The favourable effect of LV pacing on the failing heart's contractility and external constraint may help to slow the progression of disease. LV pacing also reduces the work performed by the LV as well as beat to beat transmural stress. It is possible that with chronic treatment, a small change in detrimental physiology might realize clinical benefit. Further studies are warranted to evaluate these hypotheses.

\section{Study Limitations}

We had a relatively small number of patients with low EF and mechanical dyssynchrony; all had advanced DMD associated cardiac disease (2 from group D and 7 from group E). All of the patients in group E had extensive fibrosis, obviously due to selection bias less advanced cases were missed. To prove that our analysis is valid, response to biventricular pacing would be most optimal. Tag analysis was performed up to 3rd frame after peak systole, (due to tag fading), it is possible that very dyssynchronous segments were missed, however the prevalence of mechanical dyssynchrony in boys with most advanced disease was similar to that seen in adult heart failure patients.

\section{Conclusions}

Mechanical dyssynchrony was seen in $17.1 \%$ of DMD boys with $\mathrm{EF}<55 \%$ and in $31.2 \%$ of boys with most advanced stages of disease (low EF and fibrosis); however, the observed dyssynchrony was dispersed in nature. It remains to be seen if those patients would respond to CRT.

\section{Acknowledgements}

We would like to dedicate this paper in memory of our friend and colleague William M Gottliebson M.D. We also want to thank Lisa Ambach for her assistance as secretarial support.

\section{Author details}

${ }^{1}$ The Heart Institute and Cincinnati Children's Hospital Medical Center, Cincinnati, Ohio, USA. 'Department of Radiology, Cincinnati Children's Hospital Medical Center, Cincinnati, Ohio, USA. ${ }^{3}$ Duke University School of Medicine, Durham, North Carolina, USA. ${ }^{4}$ Methodist Hospital, Houston, Texas, USA. ${ }^{5}$ The Heart and Vascular Center, The Christ Hospital, Cincinnati, Ohio, USA.

\section{Authors' contributions}

$\mathrm{KNH}, \mathrm{WM}$ and WMG contributed to all aspects of the manuscript's conception, design, data analysis, collection, critical revision and final approval. JPW, MDT, HAR, SFN, NA, ESC and DWB contributed in data analysis, critical revision and final approval of the manuscript.

\section{Competing interests}

The authors declare that they have no competing interests.

Received: 21 September 2010 Accepted: 2 February 2011

Published: 2 February 2011

References

1. Cazeau S, Leclercq C, Lavergne T, Walker S, Varma C, Linde C, et al: Effects of multisite biventricular pacing in patients with heart failure and intraventricular conduction delay. N Engl J Med 2001, 344(12):873-80.

2. Cleland JG, Daubert JC, Erdmann E, Freemantle N, Gras D, Kappenberger L, et al: The effect of cardiac resynchronization on morbidity and mortality in heart failure. N Engl J Med 2005, 352(15):1539-49.

3. Bristow MR, Saxon LA, Boehmer J, Krueger S, Kass DA, De Marco T, et al: Cardiac-resynchronization therapy with or without an implantable defibrillator in advanced chronic heart failure. N Engl J Med 2004, 350(21):2140-50.

4. Lubitz SA, Leong-Sit P, Fine N, Kramer DB, Singh J, Ellinor PT: Effectiveness of cardiac resynchronization therapy in mild congestive heart failure: systematic review and meta-analysis of randomized trials. Eur J Heart Fail 2010, 12(4):360-6.

5. van Bommel RJ, Gorcsan J, Chung ES, Abraham WT, Gjestvang FT, Leclercq C, et al: Effects of cardiac resynchronisation therapy in patients with heart failure having a narrow QRS Complex enrolled in PROSPECT. Heart 2010, 96(14):1107-13

6. Ng K, Kedia N, Martin D, Tchou P, Natale A, Wilkoff B, et al: The benefits of biventricular pacing in heart failure patients with narrow QRS, NYHA class II and right ventricular pacing. Pacing Clin Electrophysiol 2007 30(2):193-8.

7. Yu CM, Chan YS, Zhang Q, Yip GW, Chan CK, Kum LC, et al: Benefits of cardiac resynchronization therapy for heart failure patients with narrow QRS complexes and coexisting systolic asynchrony by echocardiography. J Am Coll Cardiol 2006, 48(11):2251-7.

8. Bleeker GB, Holman ER, Steendijk P, Boersma E, van der Wall EE, Schalij MJ, et al: Cardiac resynchronization therapy in patients with a narrow QRS complex. J Am Coll Cardiol 2006, 48(11):2243-50.

9. Beshai JF, Grimm RA, Nagueh SF, Baker JH, Beau SL, Greenberg SM, et al: Cardiac-resynchronization therapy in heart failure with narrow QRS complexes. N Engl J Med 2007, 357(24):2461-71.

10. Dubin AM, Janousek J, Rhee E, Strieper MJ, Cecchin F, Law $H$, et al: Resynchronization therapy in pediatric and congenital heart disease patients: an international multicenter study. J Am Coll Cardiol 2005, 46(12):2277-83.

11. Hoffman EP, Brown RH, Kunkel LM: Dystrophin: the protein product of the Duchene muscular dystrophy locus. 1987. Biotechnology 1992, 24:457-66.

12. Kunkel LM, Monaco AP, Middlesworth W, Ochs HD, Latt SA: Specific cloning of DNA fragments absent from the DNA of a male patient with an X chromosome deletion. Proc Natl Acad Sci USA 1985, 82(14):4778-82

13. Ray PN, Belfall B, Duff C, Logan C, Kean V, Thompson MW, et al: Cloning of the breakpoint of an $\mathrm{X}_{i 2} 21$ translocation associated with Duchenne muscular dystrophy. Nature 1985, 318(6047):672-5.

14. Perloff JK, de Leon AC Jr, O'Doherty D: The cardiomyopathy of progressive muscular dystrophy. Circulation 1966, 33(4):625-48.

15. Perloff JK, Roberts WC, de Leon AC Jr, O'Doherty D: The distinctive electrocardiogram of Duchenne's progressive muscular dystrophy. An electrocardiographic-pathologic correlative study. The American journal of medicine 1967, 42(2):179-88. 
16. Frankel KA, Rosser RJ: The pathology of the heart in progressive muscular dystrophy: epimyocardial fibrosis. Human pathology 1976, 7(4):375-86.

17. Sanyal SK, Johnson WW, Thapar MK, Pitner SE: An ultrastructural basis for electrocardiographic alterations associated with Duchenne's progressive muscular dystrophy. Circulation 1978, 57(6):1122-9.

18. Fornwalt BK, Arita T, Bhasin M, Voulgaris $G$, Merlino JD, Leon AR, et al: Cross-correlation quantification of dyssynchrony: a new method for quantifying the synchrony of contraction and relaxation in the heart. $J$ Am Soc Echocardiogr 2007, 20(12):1330-7 e1.

19. Gotte MJ, Germans T, Russel IK, Zwanenburg JJ, Marcus JT, van Rossum AC, et al: Myocardial strain and torsion quantified by cardiovascular magnetic resonance tissue tagging: studies in normal and impaired left ventricular function. J Am Coll Cardiol 2006, 48(10):2002-11.

20. Osman NF, Kerwin WS, McVeigh ER, Prince JL: Cardiac motion tracking using CINE harmonic phase (HARP) magnetic resonance imaging. Magn Reson Med 1999, 42(6):1048-60

21. Osman NF, McVeigh ER, Prince $\mathrm{JL}$ : Imaging heart motion using harmonic phase MRI. IEEE Trans Med Imaging 2000, 19(3):186-202.

22. Osman NF, Prince JL: Regenerating MR tagged images using harmonic phase (HARP) methods. IEEE Trans Biomed Eng 2004, 51(8):1428-33.

23. Garot J, Bluemke DA, Osman NF, Rochitte CE, McVeigh ER, Zerhouni EA, et al: Fast determination of regional myocardial strain fields from tagged cardiac images using harmonic phase MRI. Circulation 2000, 101(9):981-8.

24. Hor KN, Wansapura J, Markham LW, Mazur W, Cripe LH, Fleck R, et al: Circumferential strain analysis identifies strata of cardiomyopathy in Duchenne muscular dystrophy: a cardiac magnetic resonance tagging study. J Am Coll Cardiol 2009, 53(14):1204-10.

25. Ashford MW Jr, Liu W, Lin SJ, Abraszewski P, Caruthers SD, Connolly AM, et al: Occult cardiac contractile dysfunction in dystrophin-deficient children revealed by cardiac magnetic resonance strain imaging. Circulation 2005, 112(16):2462-7.

26. Lardo AC, Abraham TP, Kass DA: Magnetic resonance imaging assessment of ventricular dyssynchrony: current and emerging concepts. J Am Coll Cardiol 2005, 46(12):2223-8

27. Leclerca C, Faris O, Tunin R, Johnson J, Kato R, Evans F, et al: Systolic improvement and mechanical resynchronization does not require electrical synchrony in the dilated failing heart with left bundle-branch block. Circulation 2002, 106(14):1760-3.

28. Helm RH, Leclercq C, Faris OP, Ozturk C, McVeigh E, Lardo AC, et al: Cardiac dyssynchrony analysis using circumferential versus longitudinal strain: implications for assessing cardiac resynchronization. Circulation 2005, 111(21):2760-7.

29. Delfino JG, Fornwalt BK, Eisner RL, Leon AR, Oshinski JN: Cross-correlation delay to quantify myocardial dyssynchrony from phase contrast magnetic resonance (PCMR) velocity data. J Magn Reson Imaging 2008, 28(5):1086-91.

30. Silva E, Sitges M, Mont L, Delgado V, Tamborero D, Vidal B, et al: Quantification of left ventricular asynchrony throughout the whole cardiac cycle with a computed algorithm: application for optimizing resynchronization therapy. J Cardiovasc Electrophysio/ 2009, 20(10):1130-6.

31. Fornwalt BK, Thomas JA, Bhasin M, Merlino JD, Leon AR, Fyfe DA, et al: Effects of region of interest tracking on the diagnosis of left ventricular dyssynchrony from Doppler tissue images. J Am Soc Echocardiogr 2008, 21(3):234-40

32. Han Y, Chan J, Haber I, Peters DC, Zimetbaum PJ, Manning WJ, et al: Circumferential myocardial strain in cardiomyopathy with and without left bundle branch block. J Cardiovasc Magn Reson 2010, 12:2.

33. Bleeker GB, Kaandorp TA, Lamb HJ, Boersma E, Steendijk P, de Roos A, et al: Effect of posterolateral scar tissue on clinical and echocardiographic improvement after cardiac resynchronization therapy. Circulation 2006, 113(7):969-76

34. White JA, Yee R, Yuan X, Krahn A, Skanes A, Parker M, et al: Delayed enhancement magnetic resonance imaging predicts response to cardiac resynchronization therapy in patients with intraventricular dyssynchrony. J Am Coll Cardiol 2006, 48(10):1953-60.

35. Bilchick KC, Dimaano V, Wu KC, Helm RH, Weiss RG, Lima JA, et al: Cardiac magnetic resonance assessment of dyssynchrony and myocardial scar predicts function class improvement following cardiac resynchronization therapy. JACC Cardiovasc Imaging 2008, 1(5):561-8.
doi:10.1186/1532-429X-13-12

Cite this article as: Hor et al.: Presence of mechanical dyssynchrony in duchenne muscular dystrophy. Journal of Cardiovascular Magnetic Resonance 2011 13:12.

\section{Submit your next manuscript to BioMed Central and take full advantage of:}

- Convenient online submission

- Thorough peer review

- No space constraints or color figure charges

- Immediate publication on acceptance

- Inclusion in PubMed, CAS, Scopus and Google Scholar

- Research which is freely available for redistribution 